\title{
First Application of an InfraRed Imaging Video Bolometer to Heliotron J Plasma
}

\author{
Akira MIYASHITA, Kiyofumi MUKAI ${ }^{1,2)}$, Shinsuke OHSHIMA ${ }^{3)}$, Ryota MATOIKE, \\ Byron J. PETERSON ${ }^{1,2)}$, Shinji KOBAYASHI ${ }^{3)}$, Hiroyuki OKADA ${ }^{3)}$, Shinichiro KADO ${ }^{3)}$, \\ Takashi MINAMI $^{3)}$, Tohru MIZUUCHI ${ }^{3)}$, Shigeru KONOSHIMA ${ }^{3)}$ and Kazunobu NAGASAKI ${ }^{3)}$ \\ Graduate School of Energy Science, Kyoto University, Kyoto 606-8501, Japan \\ ${ }^{1)}$ National Institute for Fusion Science, Toki 509-5292, Japan \\ ${ }^{2)}$ The Graduate University for Advanced Studies, SOKENDAI, Kanagawa 240-0193, Japan \\ ${ }^{3)}$ Institute of Advanced Energy, Kyoto University, Kyoto 611-0011, Japan
}

(Received 12 March 2021 / Accepted 20 April 2021)

\begin{abstract}
The spatial radiation distribution has been measured with an infrared imaging video bolometer (IRVB) in a neutral beam injected (NBI) plasma from the Heliotron J device. The temperature distribution on the IRVB foil is consistent with radiation simulated by the three-dimensional transport code, EMC3-EIRENE. The foil temperature increase is proportional to the radiation intensity measured with an Absolute eXtreme UltraViolet (AXUV) diode diagnostic. These results suggest that the IRVB can be used for plasma radiation measurements in small and medium size devices.
\end{abstract}

(c) 2021 The Japan Society of Plasma Science and Nuclear Fusion Research

Keywords: radiation, imaging bolometer, IRVB, AXUV, Heliotron J

DOI: $10.1585 /$ pfr.16.1202079

Measurement of the spatial distribution of plasma radiation is an important issue for understanding the physics of thermal and impurity transport and the detached divertor and radiative collapse in magnetically confined plasmas. Multi-dimensional measurement is required to obtain the plasma radiation distribution in three-dimensional plasmas, such as stellarator/heliotron plasmas. The infrared imaging video bolometer (IRVB) [1] has been recognized as a two-dimensional radiation diagnostic. The purpose of this study is to apply the IRVB to an advanced helical device, Heliotron $\mathrm{J}$ [2], and to demonstrate its capability for radiation distribution measurement in small and medium size devices.

A schematic of the IRVB system installed on Heliotron $\mathrm{J}$ is shown in Fig. 1. The IRVB system is a type of pinhole camera, comprised of a pinhole $(5 \mathrm{~mm} \times$ $5 \mathrm{~mm})$, a thin foil $(35 \mathrm{~mm} \times 35 \mathrm{~mm}$ wide and $2.5 \mu \mathrm{m}$ thick, made from platinum and coated with carbon on both sides), and an infrared (IR) camera (DIAS infrared GmbH/PYROVIEW 640Lc, $50 \mathrm{fps}$, noise equivalent temperature difference: NETD $<0.08 \mathrm{~K}$ ). The plasma radiation passing through the pinhole is imaged on the foil. The foil temperature increases in response to the radiation and the IR camera measures the temperature distribution on the foil from the side opposite the plasma. The radiation distribution is reconstructed from the temperature distribution measured by the IR camera by solving the twodimensional thermal diffusion equation [1].
The IRVB observes the plasma from the outside of the torus, as shown in Fig. 1. The viewing field angles cover the plasma from the plasma core to the edge. The region outside the last closed flux surface is also measured to evaluate the background noise of the IRVB signal. In total, 49 channels are used, with each channel having a $5 \mathrm{~mm} \times 5 \mathrm{~mm}$ square area. A spatial resolution of $3.5 \mathrm{~cm}$ is obtained at the major radius, $R=1.2 \mathrm{~m}$, through the magnetic axis. A simulation result of the radiation distribution is shown in Fig. 1 (c), modeled by the three-dimensional transport code EMC3-EIRENE [3], which is projected on the surface at the same position as the IRVB foil.

IRVB measurements were performed in a neutral beam injected (NBI) plasma on the Heliotron $\mathbf{J}$ research device. A reproducible signal variation, induced by magnetic field generation, was observed. This variation was removed by subtracting data acquired with only the magnetic field on. Figure 2 shows a typical spatial and temporal low-pass filtered temperature distribution increase from the background and only on the foil, $\Delta T$. The temperature increase is observed during the discharge from the top to the lower right region of the foil while the temperature in the lower left does not show any response. This is as expected from the modeling shown in Fig. 1 (c). The upper region views the plasma core and has a larger $\Delta T$ increase than the other regions. The Absolute eXtreme UltraViolet (AXUV) intensity also has larger signals in the line of sight, where the plasma core is viewed $[4,5]$. Although the EMC3-EIRENE calculation result should not be directly 


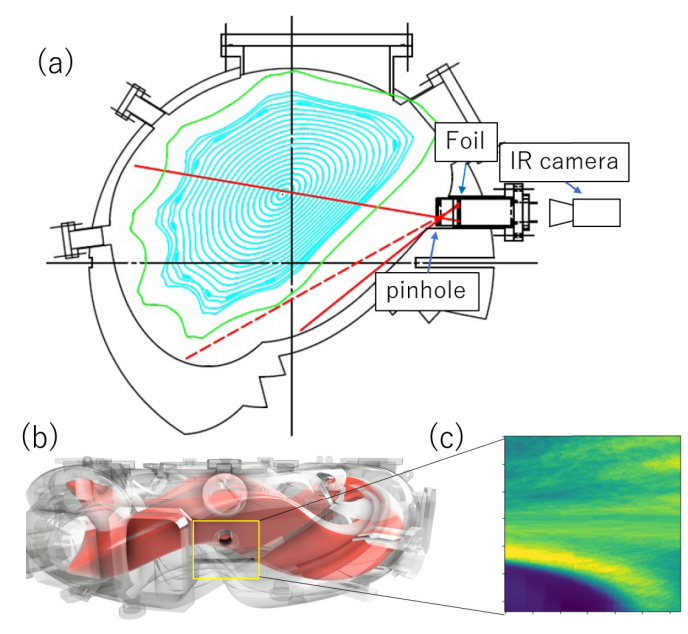

Fig. 1 (a) Poloidal cross-section of flux surfaces (blue), the region where the plasma is known to exist (green), and the viewing angle of IRVB (red). No radiation is expected at the area below the red dashed line. (b) Side view of a Heliotron J CAD figure and (c) radiation distribution simulated with EMC3-EIRENE.

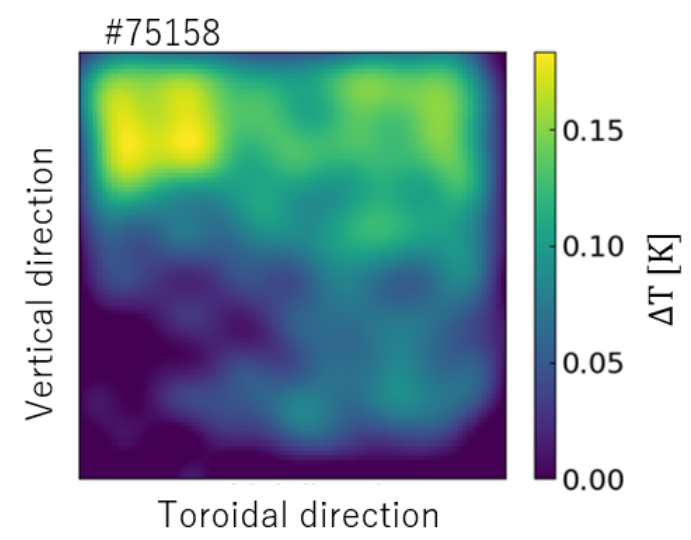

Fig. 2 Spatial distribution of temperature increase measured with IRVB in NBI plasma.

compared to the IRVB results, due to the exclusion of radiation from the core, no response is found in the foil region with no plasma, as expected in the EMC3-EIRENE modeling.

The time evolution of the temperature on the foil is shown in Figs. 3 (a) and (b) with heating power from the magnetron and NBI. Here, the blue and orange lines are the $\Delta T$ averaged in the upper-half and lower-left regions of the foil, respectively. A seed plasma is produced with the assistance of $2.45 \mathrm{GHz}$ non-resonant pre-heating and a high-temperature plasma is produced using NBI [6]. The $\Delta T$ gradually increases in the upper region at the timing of the magnetron turn-on and a sharp increase in $\Delta T$ is observed immediately after NBI injection. The magnetron is turned off at the same time as NBI injection, and hence only the plasma radiation should contribute to the $\Delta T$ re-

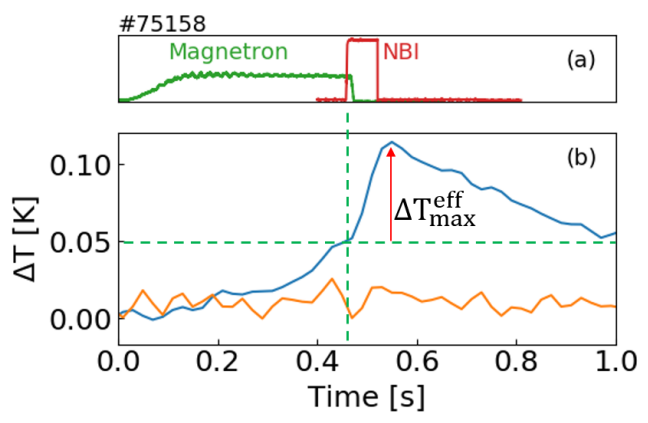

Fig. 3 Time evolution of (a) magnetron and NBI power, and (b) the temperature of the foil. In (b), the blue and orange lines show the temperature increase in the upper and lower-left regions of the foil, respectively.

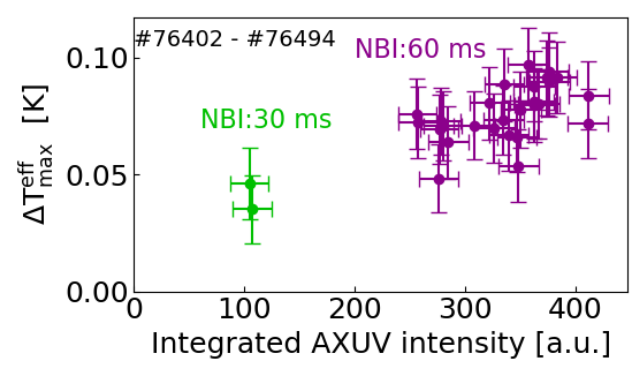

Fig. 4 Comparison of the effective temperature increase in the foil with the integrated intensity of the AXUV. The offset of the temperature increase induced by the magnetron is considered.

sponse. The $\Delta T$ in the lower left (no plasma) region is kept around zero when the $\Delta T$ in the upper region increases. The maximum of the $\Delta T$ due to the plasma radiation, $\Delta T_{\max }^{\mathrm{eff}}$, is $0.05 \mathrm{~K}$. Note that pixel and time averaging are applied to reduce the NETD.

The $\Delta T_{\max }^{\mathrm{eff}}$ is compared with the AXUV intensity in Fig. 4. The AXUV diode array with 16 channels measures the plasma radiation from the magnetic axis to the edge region. The AXUV signal is temporally integrated because the integrated value is related to the $\Delta T$, and is spatially integrated to account for the effect of thermal diffusion in the foil. The error bars represent the standard deviation of the background signal level, estimated from data without plasma. Although the sensitivity of the AXUV depends on the wavelength of radiation [5,7], it is proportional to the AXUV intensity. These results suggest that the IRVB can be used for plasma radiation measurements in small and medium size devices.

A new thinner foil, using different materials such as titanium, is under development since improvement of the IRVB sensitivity is required to measure spatial plasma radiation.

This work was supported by JSPS Core-to-Core Program, A. Advanced Research Networks, "PLADyS", JSPS KAKENHI Grant No. JP19K14689, and NIFS/NINS 
Grant No. NIFS10KUHL030 and No. NIFS19KUHL089.

[1] B.J. Peterson et al., Rev. Sci. Instrum. 79, 10E301 (2008).

[2] T. Obiki et al., Nucl. Fusion 41, 833, (2001).

[3] R. Matoike et al., Plasma Fusion Res. 14, 3403127 (2019).
[4] K. Mukai et al., Plasma Fusion Res. 6, 1402111 (2011).

[5] R.L. Boivin et al., Rev. Sci. Instrum. 70, 260 (1999).

[6] S. Kobayashi et al., Nucl. Fusion 51, 062002 (2011).

[7] M.L. Reinke and I.H. Hutchinson, Rev. Sci. Instrum. 79, 10F306 (2008). 